\title{
Dossier
}

\author{
Ancrages politiques \\ Coordonné par Jean-Louis Briquet et Élise Massicard
}




\section{Les machines politiques aux États-Unis. Clientélisme et immigration entre 1870 et $1950^{1}$}

François Bonnet

Résumé - Les machines politiques sont un phénomène lié à l'immigration irlandaise aux États-Unis entre les années 1870 et 1950. Les machines sont des organisations conçues pour gagner les élections en mobilisant des clientèles dans le cadre de relations personnelles et de solidarités ethniques. La mobilisation des clientèles passe notamment par la distribution d'emplois publics. Le débat historiographique porte sur le rôle joué par les machines auprès des immigrés pauvres. Le cas des machines montre l'intérêt que revêt le clientélisme pour étudier les trajectoires spécifiques d'intégration des groupes immigrés dans la société américaine.

1. Ce travail a fait l'objet d'un financement de la Chaire Villes de Sciences Po et a bénéficié du soutien matériel du Centre d'études européennes et de l'Observatoire français des conjonctures économiques à Sciences Po. Il a également bénéficié des conseils de J.-L. Briquet, D. Lorrain et C. Théry. 


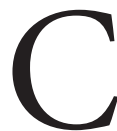

e texte porte sur les machines politiques aux États-Unis de 1870 à 1950, une forme singulière d'ancrage social des partis politiques. Les machines sont des organisations politiques spécialisées dans le clientélisme. « Machine » n'est pas synonyme de " clientélisme » : le politiste Raymond Wolfinger propose de distinguer le clientélisme (machine politics) des machines politiques (political machines) ${ }^{2}$. Le clientélisme est consubstantiel à la politique, à moins d'imaginer un monde social "glacé », selon l'expression de Jean-Louis Briquet et Frédéric Sawicki ${ }^{3}$, tandis que les machines politiques (des organisations centralisées qui dominent la vie politique locale) sont un phénomène relativement spécifique aux États-Unis des années 1870-1950.

La machine politique est une organisation conçue pour gagner les élections en mobilisant des clientèles dans le cadre de relations personnelles et de solidarités ethniques. Les machines ne cherchent pas à mobiliser leur électorat en fonction de son intérêt, de ses valeurs ou d'une idéologie ; la fidélisation des clientèles repose sur la distribution d'incitations matérielles, notamment des emplois publics. L'enjeu de la compétition politique est le contrôle de la distribution des biens clientélaires. La raison d'être des machines politiques est donc de former des coalitions électorales durables dans un contexte démographique instable de manière à sécuriser les ressources du gouvernement urbain ; or, typiquement, le vote sur les idées (issue voting) est trop « volatile » ${ }^{4}$. Dennis William Brogan, un politiste écossais spécialiste des États-Unis, résume ce phénomène : «La nature profonde de la machine est son indifférentisme politique. Elle existe pour elle-même ${ }^{5}$.»

Le développement des machines s'inscrit dans la trajectoire particulière de la polity américaine. Au contraire des vieux États européens, où la centralisation du pouvoir et le développement de l'administration précèdent la démocratisation, la démocratisation survient aux États-Unis avant le développement de la bureaucratie ${ }^{6}$. En principe, la logique de la bureaucratie est précisément de limiter au maximum ce qui définit la relation de clientèle, à savoir la prégnance des relations personnelles et des intérêts particuliers, le primat de la loyauté sur les règlements, le primat de la réciprocité sur le contrat. La bureaucratie fonctionne selon des règles, et refuse, par principe, toute dérogation au règlement et a fortiori toute faveur personnelle, toute exception pour les situations

2. Wolfinger (R.), "Why Political Machines Have Not Withered Away and Other Revisionist Thoughts ", Journal of Politics, 34 (2), 1972.

3. Briquet (J.-L.), Sawicki (F.), dir., Le clientélisme politique dans les sociétés contemporaines, Paris, PUF, 1998, p. 2. Sur le clientélisme en général, cf. Médard (J.-F.), « Le rapport de clientèle. Du phénomène social à l'analyse politique ", Revue française de science politique, 26 (1), 1976 ; Briquet (J.-L.), « La politique clientélaire. Clientélisme et processus politiques ", in Briquet (J.-L.), Sawicki (F.), dir., Le clientélisme politique, op. cit.

4. Stone (C.), «Urban Political Machines: Taking Stock », PS: Political Science and Politics, 29 (3), 1996.

5. Cité in Banfield (E. C.), Wilson (J. Q.), City Politics, Cambridge (Mass.), Harvard University Press/MIT Press, 1963, p. 116. Cette citation, comme celles qui suivent, est traduite par l'auteur.

6. Shefter (M.), Political Parties and the State, Princeton, Princeton University Press, 1994. 
particulières ${ }^{7}$. La relation de clientèle repose au contraire sur les faveurs, les recommandations personnelles, l'amitié, la corruption, la solidarité identitaire. L'instauration du suffrage « universel » (blanc et masculin) a lieu alors que la nation américaine est en formation et les États fédérés sont jaloux de leurs prérogatives. Le déploiement d'institutions démocratiques en l'absence de bureaucratie légale-rationnelle permet le développement du spoils system : les emplois publics sont attribués par le vainqueur de l'élection, pour la durée de la mandature. Il est significatif qu'Edward Banfield et James Q. Wilson énoncent à la première page de City Politics que tout ce qu'a pu écrire Max Weber sur la bureaucratie et l'État moderne ne s'applique pas au cas américain ${ }^{8}$.

Ce travail, qui repose sur des sources secondaires, entend faire le point des connaissances sur le fonctionnement des machines politiques aux États-Unis, ainsi que resituer le débat historiographique sur le caractère progressiste ou non des machines. L'analyse des machines permet un utile changement de perspective dans la sociologie française du clientélisme, qui a tendance à s'intéresser au clientélisme comme catégorie savante de délégitimation de pratiques politiques populaires. Le cas des machines américaines montre l'intérêt que revêt le clientélisme pour étudier les trajectoires spécifiques d'intégration des groupes immigrés dans la société américaine.

\section{L'ancrage social des machines politiques}

\section{Machines et immigration irlandaise}

L'émergence des machines est liée à l'urbanisation et à l'immigration. Entre 1830 et 1930, la population américaine passe de 12 à 130 millions d'habitants, et plus de 30 millions d'immigrés s'installent aux États-Unis. La plupart des immigrés se concentrent dans les villes côtières et au bord des grands lacs, qui connaissent une croissance spectaculaire. Sur la période, New York passe de 240000 à 7 millions d'habitants, Chicago de 4500 à 2,7 millions. À Chicago, en 1900, la moitié de la ville est née à l'étranger 9 . Une des conséquences de l'immigration dans la vie politique américaine est le brouillage du clivage de classe et l'affaiblissement du mouvement ouvrier ${ }^{10}$. Dans le Nord-Est et dans les villes

7. Weber (M.), Économie et société, Paris, Pocket, « Agora », 1995 [1 1 re éd. 1922].

8. Banfield (E.C.), Wilson (J. Q.), City Politics, op. cit., p. 1.

9. Lors de son voyage en Amérique, Weber (qui prépare l'Éthique protestante) décrit Chicago comme une ville " ahurissante ", immense, avec des gargotes chinoises, juives, allemandes, polonaises, grecques, croates, des immenses abattoirs, des gratte-ciels et des prostituées en vitrine. Cf. Chapoulie (J.-M.), La tradition sociologique de Chicago 1892-1961, Paris, Seuil, 2001 ; Garant (N.), Larochelle (G.), « Weber en Amérique. Le crépuscule de l'esprit du capitalisme ", Revue internationale de politique comparée, 10 (4), 2003.

10. L'immigration n'en est pas la seule cause : la conquête de l'Ouest a offert une stratégie d'exit aux démunis à la recherche d'opportunités, tandis que la pratique de l'esclavage dans le Sud et le racisme ont nourri une " identité blanche ». Cf. Amenta (E.), Bonastia (C.), Caren (N.), « US Social Policy in Comparative and Historical Perspective: Concepts, Images, Arguments, and Research Strategies », Annual Review of Sociology, 27,2001 . 
du Midwest, les conflits entre les différents groupes immigrés contribuent à empêcher le développement d'une solidarité de classe. Au lieu de développer un mouvement ouvrier comme leurs homologues européens, les classes populaires américaines s'opposent entre Américains et immigrés, entre immigrés catholiques et immigrés protestants, entre Irlandais et Italiens, entre Slaves et Juifs.

Ces divisions structurent les partis politiques au niveau national au XIX ${ }^{e}$ siècle. Les Républicains tendent à représenter les Américains « de souche » (Anglais et Hollandais), les immigrés protestants (Allemands et Scandinaves), les ruraux, le Midwest et l'État fédéral, tandis que les Démocrates tendent à représenter les immigrés catholiques, les urbains, le Sud et les États fédérés. Les partis politiques ne sont pas structurés selon les classes sociales, il n'y a donc pas de parti socialiste ou social-démocrate pour revendiquer des droits sociaux et le Parti démocrate n'a pas de fondement idéologique socialiste, contrairement aux partis de gauche européens ${ }^{11}$. Les politiques sociales ne sont donc pas organisées pour les workers (comme en Europe) mais pour les mothers and soldiers, selon le mot de Theda Skocpol ${ }^{12}$. Alors qu'en Europe se développent les systèmes d'assurance ou de redistribution, les politiques sociales aux États-Unis sont le fait des associations, des Églises, de la charité locale et du clientélisme des gouvernements urbains ${ }^{13}$. Les électeurs américains des classes populaires ne sont pas dans un jeu où la mobilisation politique de la conscience de classe est porteuse de progrès social. Le Parti démocrate ne vise pas à prendre le pouvoir au niveau national pour accaparer les ressources de l'État et défendre les intérêts de la classe ouvrière, mais à gagner les élections locales pour contrôler la distribution des ressources des gouvernements urbains afin de gagner les élections suivantes. Les partis impliqués dans le clientélisme n'ont pas intérêt à faire émerger une bureaucratie professionnelle qui les privera de leur pouvoir discrétionnaire dans la distribution des emplois et des ressources ${ }^{14}$.

La distribution des ressources par la voie clientélaire, c'est-à-dire selon des solidarités ethniques, contribue à la dissociation de la conscience de classe de la compétition politique. Les machines urbaines américaines sont principalement le fait de politiciens irlandais, en lien avec des clientèles irlandaises, dans le cadre du Parti démocrate ${ }^{15}$. Les Irlandais sont pauvres ; la première

11. Moore (B.), Social Origins of Democracy and Dictatorship. Lord and Peasant in the Making of the Modern World, Boston, Beacon Press, 1966 ; Katznelson (I.), Zolberg (A.), Working Class Formation, Princeton, Princeton University Press, 1986.

12. Skocpol (T.), Protecting Soldiers and Mothers: The Political Origins of Social Policy in the United States, Cambridge, Belknap Press of Harvard University Press, 1992.

13. Ibid. Cf. également Skocpol (T.), «Formation de l'État et politiques sociales aux États-Unis », Actes de la recherche en sciences sociales, 96-97, 1993.

14. Mayhew (D.), Placing Parties in American Politics, Princeton, Princeton University Press, 1986.

15. Erie (S.), Rainbow's End. Irish-Americans and the Dilemmas of Urban Machine Politics, 1840-1985, Berkeley, University of California Press, 1988. Sur la période étudiée (1870-1950), il existe quelques machines urbaines républicaines isolées (Rochester, Cincinnati, Philadelphie). Les Républicains, grâce à leur implantation rurale, contrôlent la plupart des États comme gouverneurs. 
génération fuit la grande famine en Irlande (un million de morts entre 1846 et 1851). Les immigrés pauvres forment la clientèle naturelle des machines : ils sont naturellement plus sensibles aux incitations matérielles que constituent les biens clientélaires que les classes moyennes, et ils sont un bien abondant dans les villes américaines. L'explication de la prépondérance des Irlandais dans le phénomène clientélaire américain est un sujet en soi ${ }^{16}$. Ce qui importe ici est de souligner le caractère ethnique de la machine, à la fois dans l'allocation des ressources (les Irlandais sont les principaux bénéficiaires) et dans la formation des clivages politiques urbains. Les machines reposant sur l'interconnaissance et les rapports de réciprocité, elles mobilisent à la fois selon des logiques de parenté, de voisinage et de monde professionnel (à travers les syndicats), mais toujours de manière préférentielle au sein du groupe ethnique ${ }^{17}$.

\section{L'organisation des machines politiques et la distribution des biens clientélaires}

À la tête de la machine se trouve le boss. Le boss est toujours le chef du parti ; il contrôle le processus de nomination et a autorité sur ses lieutenants au niveau des différents quartiers. Il peut être aussi maire de la ville, comme Richard J. Daley, le boss de Chicago, qui a été maire pendant 21 ans, Frank Hague à Jersey City ou Dan O'Connell à Albany. Mais il peut rester un personnage discret dans la vie publique, nommant des candidats fantoches aux élections et délaissant les aspects cérémoniaux de la fonction municipale pour se consacrer à l'exercice du pouvoir, comme les «sachems » de Tammany à New York ou Ed Crump à Memphis (cf. tableau 1).

La figure du boss ne doit pas occulter la complexité de l'organisation territoriale de la machine. Chaque ville est divisée en circonscriptions, dont le plus petit niveau est le precinct. Alors que les plus petites circonscriptions des villes européennes sont constituées d'au moins 2000 électeurs, les circonscriptions des villes américaines totalisent environ 400 électeurs. Il est donc humainement possible pour un politicien (certes doué pour les relations humaines) de connaître personnellement tous les électeurs d'une circonscription ${ }^{18}$. Ce politicien, le precinct captain, est l'homme de base de la machine ; il est sélectionné sur ses capacités à mobiliser les électeurs. Il n'exerce pas de fonction élective rémunérée ; la machine lui fournit un emploi municipal en fonction de ses performances, mesurées aux élections. Les meilleurs precinct captains peuvent

16. Ibid., p. 31-32, pour un examen des explications disponibles.

17. À Chicago par exemple, les vieux amis de R. J. Daley (cf. tableau 1) dans le quartier de Bridgeport étaient favorisés par rapport aux autres Irlandais, mais la fonction première de la machine était d'assurer aux Irlandais un meilleur statut qu'aux Polonais ou aux Italiens. Cf. Royko (M.), Boss. Richard J. Daley of Chicago, New York, Plume, 1988 [1 $1^{\text {re }}$ éd. 1972].

18. Gosnell (H.), «The Political Party versus the Political Machine », Annals of American Academy of Political and Social Science, 169, 1933. 
grimper dans la hiérarchie et s'occuper d'un ward ; s'ils sont mécontents, ils peuvent décider de travailler pour une faction concurrente. Un ward regroupe 30 à 40 precincts (environ 15000 électeurs) et le ward leader est un personnage politique significatif : il est souvent conseiller municipal. Le ward leader occupe une position intermédiaire dans la hiérarchie : il est responsable de la sélection des precincts captains et rend compte au boss.

Tableau 1. Récapitulatif des boss et des machines cités

\begin{tabular}{|l|l|l|}
\hline \multicolumn{1}{|c|}{ Ville } & \multicolumn{1}{|c|}{ Boss } & \multicolumn{1}{c|}{ Années } \\
\hline \multirow{2}{*}{$\begin{array}{l}\text { New York } \\
\text { (Tammany Hall) }\end{array}$} & $\begin{array}{l}\text { William M. Tweed } \\
\text { (Boss Tweed) }\end{array}$ & $\begin{array}{l}\text { «Grand Sachem » de Tammany } \\
\text { Hall : 1858-1871 }\end{array}$ \\
\cline { 2 - 3 } & $\begin{array}{l}\text { Richard Croker, John Kelly, } \\
\text { Charles Murphy }\end{array}$ & Années 1890-1933 \\
\hline $\begin{array}{l}\text { Chicago } \\
\text { (Cook County } \\
\text { Democratic Party) }\end{array}$ & Richard J. Daley & $1955-1976$ \\
\hline $\begin{array}{l}\text { Albany } \\
\text { (County Democra- } \\
\text { tic Committee })\end{array}$ & Dan O'Connell & $1921-1977$ \\
\hline $\begin{array}{l}\text { Kansas City } \\
\text { (Jackson County } \\
\text { Democratic Club) })\end{array}$ & Jomes Pendergast & Années 1890-1900 \\
\cline { 2 - 4 } Boston & James Michael Curley & $\begin{array}{l}\text { Quatre mandats de maire: } \\
1914-1918,1922-1926,1930- \\
1934,1946-1950\end{array}$ \\
\hline Jersey City & Frank Hague & $1917-1947$ \\
\hline Memphis & Ed Crump & $\begin{array}{l}\text { Maire : 1910-1915, boss jusqu’à } \\
\text { la fin des années 1940 }\end{array}$ \\
\hline Providence & Buddy Cianci & $1975-1984,1991-2002$ \\
\hline Cincinnati & George « Boss » Cox & Années 1880-1916 \\
\hline
\end{tabular}

L'aspect caractéristique de la machine est la centralité du clientélisme dans l'activité du parti. Les biens clientélaires par excellence que les machines échangent contre la loyauté électorale sont les emplois publics ${ }^{19}$. Les machines offrent plusieurs types d'emplois : des emplois réels, pour lesquels les salariés reçoivent

19. Ibid. Cf. également Banfield (E. C.), Wilson (J. Q.), City Politics, op. cit. ; Mladenka (K. R.), « The Urban Bureaucracy and the Chicago Political Machine: Who Gets What and the Limits to Political Control », American Political Science Review, 74 (4), 1980. 
leur salaire en échange de leur travail de pompier, policier, éboueur, assistante sociale, secrétaire, etc., et les no-shows, les emplois fictifs, pour lesquels l'employé fait par exemple officiellement partie de telle administration, mais travaille pour le compte de la machine. Outre les emplois municipaux directs, la machine peut créer de l'emploi clientélaire avec les marchés publics, en attribuant les contrats aux entreprises qui savent recruter avec discernement, c'està-dire dans l'intérêt électoral ou organisationnel de la machine ${ }^{20}$. Les emplois sont naturellement distribués dans le bassin d'électeurs dont il faut entretenir la loyauté. Jersey City, la ville de Boss Hague, a dans les années 1930 et 1940 le plus haut taux d'emploi public des grandes villes américaines. En 1970, la ville de Chicago dispose de 30000 emplois à distribuer sur un mode clientélaire, ce qui permet de conforter la position de la machine de Richard J. Daley, laquelle ponctionne $5 \%$ du salaire de chacun des employés municipaux en échange de la sécurité de l'emploi. Entre 1900 et 1930 à New York et à Albany (la capitale de l'État de New York), les deux tiers des emplois municipaux sont occupés par des Irlandais (100 000 emplois au total) ${ }^{21}$.

Dans le type idéal de la machine, Precinct captains et ward leaders sont soumis à la même contrainte de performance : ils doivent chacun estimer au plus près le nombre de votes qu'ils sont susceptibles de mobiliser le jour des élections. L'estimation qu'ils font doit être la plus juste possible. Si un precinct captain fait une estimation trop modérée, son ward leader voudra le remplacer par un rival qui se présente comme plus ambitieux. S'il promet un nombre trop important de votes et déçoit les attentes le jour des élections, il signale son incompétence et son manque de fiabilité, risque de perdre son emploi clientélaire et sera remplacé pour l'élection suivante ${ }^{22}$. Le niveau des ressources clientélaires que lui accorde le ward leader dépend de l'ambition de l'estimation et de la fiabilité de ses estimations. Le precinct captain doit donc travailler son precinct tous les jours pour s'assurer de la mobilisation de ses électeurs. Le ward leader est dans la même situation par rapport au boss : corriger les estimations de ses precinct captains pour une meilleure fiabilité, justifier sa dotation en ressources clientélaires à distribuer entre les precinct captains, pour que la machine l'emporte dans la marge désirée le jour des élections ${ }^{23}$. Les ward leaders et les precinct captains les plus loyaux, les plus efficaces et les plus fiables reçoivent plus d'emplois à distribuer, de façon à minimiser la probabilité de gaspillage des ressources. Le problème est que la machine n'a jamais assez d'emplois pour satisfaire toutes les demandes : les électeurs sont trop nombreux, les emplois trop rares, et selon le

20. Stone (C.), « Urban Political Machines... », art. cité.

21. Judd (D. R.), Swanstrom (T.), City Politics. Private Power and Public Policy, New York, Longman, 2004.

22. Johnston (M.), "Patrons and Clients, Jobs and Machines: A Case Study of the Uses of Patronage ", American Political Science Review, 73 (2), 1979.

23. Ibid. 
mot de Banfield et Wilson, il n'y a pas assez de « sauce » pour tout le monde ${ }^{24}$. La rareté des ressources incite la machine à multiplier les emplois peu qualifiés et mal payés. Gérer la rareté consiste surtout à ne donner les emplois qu'aux militants les plus dynamiques, ceux qui vont participer aux campagnes électorales, afin de ne garder les incitations moins conséquentes que pour les électeurs indécis ${ }^{25}$. Enfin, les machines ne disposent pas que d'incitations positives, mais aussi de sanctions pour les groupes qui votent «mal» : le refus de permis de construire, les tracasseries administratives pour les commerces, voire le strict respect des Sunday laws (lois interdisant le travail le dimanche) pour les Juifs, qui du coup ne peuvent travailler que cinq jours par semaine ${ }^{26}$.

L'idée de mécanique bien huilée qui découle de la métaphore de la «machine » imperturbable et parfaitement rationnelle pourrait donner l'impression d'une organisation simple et naturelle dans laquelle politiciens et électeurs échangent emplois et voix comme sur un marché. De nombreux auteurs ont montré que les machines n'ont évidemment pas un pouvoir illimité sur l'attribution des emplois publics, du fait de la bureaucratie et des contraintes techniques et professionnelles ${ }^{27}$. Un même homme politique ne peut pas courtiser des clientèles différentes dans le temps sous peine de paraitre déloyal et incohérent. Il y a donc une rigidité dans l'allocation des ressources qui est potentiellement nuisible à la machine, puisque celle-ci peut attribuer des emplois dont le potentiel électoral n'est pas optimal si la sociologie de la circonscription évolue. Leur allocation discriminatoire peut avoir pour conséquence l'exacerbation du ressentiment des électeurs des autres groupes ethniques. Avec le temps, les politiciens tendent à récompenser à outrance leurs circonscriptions initiales au-delà du bénéfice réel qu'ils peuvent escompter : les machines sont comme toutes les organisations, elles vieillissent, deviennent moins compétitives, moins efficaces, enserrées dans un réseau de contraintes issues de leurs succès passés ${ }^{28}$.

\footnotetext{
24. Banfield (E. C.), Wilson (J. Q.), City Politics, op. cit.

25. On imagine aisément que d'autres logiques sont venues parasiter la distribution rationnelle des emplois, fondées sur l'importance des relations personnelles, par exemple le népotisme. Cf. Stone (C.), « Urban Political Machines... ", art. cité.

26. Cf. Erie (S.), Rainbow's End..., op. cit.

27. Mladenka (K. R.), "The Urban Bureaucracy and the Chicago Political Machine ", art. cité ; cf. aussi Levy (F.), Meltsner (A. J.), Wildavsky (A.), Urban Outcomes, Berkeley, University of California Press, 1974 ; Lineberry (R.), Equality and Urban Policy: The Distribution of Municipal Public Services, Beverly Hills, Sage, 1977 ; Jones (B. D.) et al., « Service Delivery Rules and Distribution of Local Goverment Services: Three Detroit Bureaucracies », Journal of Politics, 40, 1978 ; Mladenka (K. R.), Hill (K. Q.), « The Distribution of Urban Police Services », Journal of Politics, 40, 1978 ; Jones (B. D.), « Party and Bureaucracy: The Influence of Intermediary Groups on Urban Public Service Delivery », American Political Science Review, 75, 1981.

28. Cf. également dans le cas de Chicago : Rakove (M.), Don't Make No Waves... And Don't Back No Losers : An Insider Analysis of the Daley Machine, Bloomington, Indiana University Press, 1975.
} 


\section{L'importance des relations personnelles}

Les machines ne sont pas qu'organisation et calcul ; elles reposent sur des relations personnelles. Il faut essayer de redonner leur juste place à ces relations personnelles qui rentrent mal dans le cadre des sciences sociales : l'amitié, la complicité, la chaleur humaine et l'effervescence des campagnes. La vie quotidienne des precinct captains et de ceux qui aspirent à le devenir est faite de rencontres dans des clubs, des pique-niques, des pubs, des banquets, partout où les gens vont boire, danser et s'amuser, aux matches de boxe et de base-ball ${ }^{29}$. Le boss a le contact facile, des manières simples, le sens des relations humaines. Il ne cherche pas à convaincre des électeurs génériques du bien-fondé de son programme, mais à être assez sympathique pour que les voisins votent pour lui - et n'hésitent pas à lui demander une faveur. George Washington Plunkitt raconte que, lorsqu'il voit un jeune homme qui a envie d'être chanteur, il se débrouille pour le faire chanter dans un banquet ou dans une église, " et il sera un supporter de Tammany pour la vie ». Si Plunkitt repère un joueur de baseball, il le fait prendre dans une équipe, « et il collera des affiches à la prochaine élection ${ }^{30}$. De fait, Plunkitt passe ses journées à aller voir des gens, à régler des petits problèmes, toujours affable, connu personnellement de milliers de NewYorkais. Buddy Cianci, le maire de Providence, fait l'objet d'un jeu de mots difficilement traduisible qui illustre cette sociabilité politique frénétique : «Buddy Cianci would jump at the opening of an enveloppe ${ }^{31}$. " Frank Hague, le boss de Jersey City, a commencé sa carrière comme manager d'un "club », en fait une bande de gros bras payés par la machine pour intimider les adversaires politiques. Ed Crump, de Memphis, passe son enfance et son adolescence dans la pauvreté des petits Blancs du Sud. Les boss les moins scrupuleux n'hésitent pas à publiciser les coups de main qu'ils donnent à leurs amis les moins fréquentables pour bien montrer qu'ils font passer l'amitié avant la probité publique. James Michael Curley (de Boston) purge deux mois en prison pour avoir passé un examen d'entrée à la poste à la place d'un sympathisant; il en retire une grande popularité. Pour aider un ami qui faisait des chèques en bois, Boss Hague de Jersey City commet un faux témoignage qui lui vaut des poursuites judiciaires mais qui fait grosse impression auprès de la communauté irlandaise.

La centralité de la sociabilité et des relations personnelles dans le fonctionnement des machines se manifeste dans la question de l'alcool. Dans le monde social des ouvriers américains, le pub (irlandais) est un lieu stratégique des quartiers populaires : dans les grandes villes, on compte jusqu'à un pub pour cinquante habitants ${ }^{32}$. C'est un espace de sociabilité pour tous ceux qui aspirent

\footnotetext{
29. Stone (C.), « Urban Political Machines... », art. cité.

30. Riordan (W. L.), Plunkitt of Tammany Hall, New York, E. P. Dutton, 1963 [1 $1^{\text {re }}$ éd. 1905].

31. Jeu de mots en anglais sur le double sens du terme opening, ouverture et inauguration.

32. Judd (D. R.), Swanstrom (T.), City Politics..., op. cit.
} 
à une carrière politique. De nombreux precinct captains commencent leur carrière comme propriétaires de pubs ou de saloons, au plus proche des électeurs. Certains boss, comme Tom Pendergast de Kansas City ou George Cox de Cincinnati, ont travaillé comme barman dans un saloon. L'alcoolisme des immigrés est un thème majeur de la période et les débordements des ouvriers irlandais et polonais sont devenus proverbiaux. La petite bourgeoisie puritaine s'alarme des ravages de l'alcoolisme dans les classes populaires et, en 1919, les Républicains votent le Prohibition Act. Les machines démocrates défendent les intérêts des consommateurs d'alcool. Ed Crump, le boss de Memphis, ne fait tout simplement pas respecter la prohibition dans sa ville. La police a pour consigne de ne pas interférer avec les détaillants ou les consommateurs ${ }^{33}$. Tom Pendergast voit la fin de son mandat ternie par des scandales liés à sa proximité avec les gangsters qui organisaient la contrebande d'alcool à Kansas City.

\section{Historiographie des machines}

Les machines politiques ont donc été une forme particulière d'ancrage social des partis politiques, reposant sur la mobilisation électorale de groupes ethniques au niveau local et le clientélisme. Mais l'analyse de la machine, son historiographie, n'est pas moins ancrée dans un contexte social et historique.

\section{La critique initiale : les réformateurs contre les machines}

La corruption et le clientélisme provoquent des scandales qui entrainent la formation de coalitions anti-machines. Dès les années 1890, les machines sont critiquées par un mouvement réformateur (reform), parfois appelé " mouvement pour le bon gouvernement ${ }^{34}$. Le mouvement réformateur recrute dans les classes moyennes protestantes qui découvrent les scandales politiques et du monde des affaires dans les écrits des muckrackers (journalistes d'investigation) comme Lincoln Steffens ${ }^{35}$. Les réformateurs stigmatisent l'incompétence des boss en matière de gouvernement urbain, leur amateurisme en politiques publiques et leur corruption. Les réformateurs dénoncent les scandales pour faire adopter des règles électorales et institutionnelles qui soient défavorables aux machines.

Les réformateurs sont avant tout scandalisés par la corruption et la fraude électorale. Les comptes rendus des muckrackers insistent sur le fait que les

\footnotetext{
33. Biles (R.), «Ed Crump versus the Unions: The Labor Movement in Memphis During the 1930s », Labor History, 25 (4), 1984.

34. Hofstadter (R.), The Age of Reform: From Bryan to FDR, New York, Vintage, 1960 [1 $1^{\text {re }}$ éd. 1955] ; Lowi (T. J.), «Machines politiques ou bureaucraties administratives : le problème du gouvernement des villes américaines », Sociologie du travail, 4, 1969 ; Teaford (J. C.), « Finis for Tweed and Steffens: Rewriting the History of Urban Rule ", Reviews in American History, 10, 1982.

35. Steffens (L.), The Shame of the Cities, General Books LLC, 2009 [1 $1^{\mathrm{re}}$ éd. 1904].
} 
milieux d'affaires participent aux activités illégales de la machine, que ce soit en corrompant des élus pour obtenir des marchés publics, ou en finançant la machine pour être protégés de la justice. Boss Tweed détourne 30 millions de dollars entre 1868 et 1871 pour son propre compte et pour celui de Tammany, soit environ 500 millions de dollars de 2009, ce qui représente $30 \%$ du budget de la ville de New York ${ }^{36}$. Frank Hague, le boss et maire de Jersey City entre 1917 et 1947, amasse une fortune estimée à 10 millions de dollars (environ 90 millions de dollars de $2009^{37}$ ) au cours de sa carrière, alors que son salaire de maire est de 8500 dollars annuels (soit un total de 255000 dollars de revenus légitimes). À Chicago dans les années 1950, il faut payer la machine démocrate 300 dollars (environ 2500 dollars de 2009) pour prétendre à un emploi municipal. Non seulement la machine ne distribue pas des jobs aux électeurs, mais en plus le fait de corrompre un precinct captain ou ward leader n'est pas une garantie d'obtenir un emploi ; cela permet au mieux de faire partie de la liste des candidats ${ }^{38}$. Frederic C. Howe, un homme politique réformateur de l'Ohio, décrit les machines comme «le lien qui unit les criminels riches avec les criminels pauvres $»^{39}$.

Le politiste réformateur Harold Gosnell insiste sur la dimension structurelle de la relation entre la machine et le milieu en présentant un système d'incitations qui conduit tous les acteurs à agir dans un sens contraire à la probité publique : "Les partis comptent sur les contributions des hommes d'affaires qui recherchent des passe-droits, des criminels qui cherchent à se protéger des interférences des autorités, et des élus dont la loyauté envers le parti dépasse celle envers l'État $[\ldots]^{40}$.» Gosnell dénonce les liens de la machine avec le crime organisé, qui fournit des services et notamment de la main-d'œuvre pour les campagnes électorales - les machines ne dédaignent pas salarier des délinquants pour servir d' "électeurs à la chaîne » (chain voters) afin de maximiser les chances de victoire le jour des élections ${ }^{41}$. Le manque de ressources clientélaires par rapport à la demande de la clientèle conduit en effet les machines à recourir à la fraude électorale pour continuer à gagner les élections. À Albany, Dan O'Connell remporte les élections avec des majorités qui représentent les deux tiers de la population totale de la ville, enfants compris. Frank Hague est le symbole des dérives anti-démocratiques des machines. Dans les années 1920, 245 étudiants

36. Judd (D. R.), Swanstrom (T.), City Politics..., op. cit.

37. Toutes les conversions en dollars de 2009 ont été réalisées à l'aide du site MeasuringWorth : Williamson (S. H.), «Six Ways to Compute the Relative Value of a U.S. Dollar Amount, 1790 to Present », MeasuringWorth, 2009, http://www.measuringworth.com/uscompare/.

38. Gecan (M.), Going Public. An Organizer's Guide to Citizen Action, Random House, Anchor Books, 2004. 39. Cité in Boulay (H.), DiGaetano (A.), « Why Did Political Machines Disappear? », Journal of Urban History, 12 (1), 1985, p. 27.

40. Gosnell (H.), «The Political Party versus the Political Machine », art. cité, p. 27.

41. Ibid. Stone raconte une anecdote : un jour d'élections, un politicien propose cinquante cents à un électeur pour voter pour la machine. L'électeur s'empresse de marchander : «Si je n'obtiens pas un dollar, je voterai mes convictions » (Stone (C.), «Urban Political Machines... », art. cité, p. 446). 
de Princeton se portent volontaires pour surveiller la régularité du scrutin tenu à Jersey City. Des gros bras sont alors envoyés à leur rencontre ; en une heure, cinq étudiants sont si sérieusement blessés que l'opération de scrutation est annulée sur le champ. Hague ordonne en outre à la police de Jersey City d'interdire tout meeting organisé par des opposants politiques.

Le caractère systémique de la corruption dans le système clientélaire indigne les réformateurs, mais peut-être pas autant que la bienveillance avec laquelle les électeurs des machines considèrent ces scandales. Une réformatrice comme Jane Addams note, désabusée : «En fait, les cadeaux et les faveurs sont reçus assez bêtement comme une preuve de gentillesse spontanée. L'alderman est vraiment élu parce qu'il est un bon ami et un bon voisin. Il est corrompu, bien sûr, mais il n'est pas élu parce qu'il est corrompu, mais plutôt en dépit de cela. Ses mœurs sont celles de ses électeurs. Il exemplifie et exagère la figure populaire du type bien. Il a atteint ce que ses électeurs désirent secrètement ${ }^{42}$. $\mathrm{Ce}$ que Lincoln Steffens, un autre réformateur, résume en une phrase : «Tammany, c'est la corruption avec l'assentiment ${ }^{43}$. " Les réformateurs ne sont cependant pas préoccupés que de "bon gouvernement ». Ils associent les Démocrates aux trois « R », «Rum, Romanism, and Rebellion» : l'alcool (par opposition au prohibitionnisme puritain), le catholicisme et la guerre de Sécession. Parmi les organisations qui ont un lien organique avec la machine, Gosnell n'hésite pas à associer l'Église catholique à la pègre et à la presse de caniveau, en raison de son lien avec l'immigration irlandaise. L'hostilité des réformateurs aux machines est aussi une hostilité aux immigrés, tout particulièrement les immigrés irlandais, italiens, juifs et slaves. De nombreux réformateurs sont membres de la Immigration Restriction League, une organisation "nativiste ", c'est-à-dire anti-immigrés ${ }^{44}$.

Parmi les réformateurs ouvertement nativistes se distingue Edward A. Ross, l'auteur de Social Control, fondateur du département de sociologie à l'Université de Chicago, qui déclare : «Les mannequins en stuc de mauvaise qualité du Sud-Est de l'Europe ne remplacent pas vraiment les fils que n'ont pas engendrés les hommes de granit qui sont tombés à Gettysburg ou a Cold Harbor ${ }^{45}$. » La tradition sociologique de Chicago est solidement ancrée dans le mouvement réformateur. Park cite abondamment Spengler et Le déclin de l'Occident dans The City. C'est cette idéologie particulière, ce progressisme puritain des classes moyennes que Charles Wright Mills fustige en dénonçant les «pathologistes

42. Cité in Banfield (E. C.), Wilson (J.Q.), City Politics, op. cit., p. 118.

43. Cité in Stone (C.), « Urban Political Machines... », art. cité, p. 770.

44. Cf. Hofstadter (R.), The Age of Reform..., op. cit.; Banfield (E. C.), Wilson (J. Q.), City Politics, op. cit.

45. Cité in Violas (P.), "Progressive Social Philosophy: Charles Horton Cooley and Edward Alsworth Ross ", in Karier (C. J.), Violas (P.), Spring (J.), eds., Roots of Crisis: The American Education in the Twentieth Century, New York, Rand McNally, 1973, p. 54. 
sociaux $»^{46}$. L'idée d'une supériorité morale des classes moyennes protestantes sur les immigrés catholiques a perduré dans l'analyse des machines à travers notamment les travaux de deux politistes conservateurs, Edward Banfield et James Q. Wilson. City Politics poursuit une intuition de Banfield et de ses travaux sur le familialisme amoral ${ }^{47}$. Pour Banfield, le clientélisme et la corruption sont ancrés dans des structures familiales archaïques et empêchent toute résolution collective des problèmes communs. Banfield et Wilson opposent donc le private regarding ethos des classes populaires immigrées qui les conduit à voter en fonction d'amitiés, d'échanges de bons procédés et en fin de compte de leur intérêt privé, au public regarding ethos des classes moyennes réformatrices, qui font leur choix en fonction de l'intérêt général. Cette analyse met en valeur le rôle joué par la culture dans la vie politique (au détriment du pouvoir ou des inégalités) et valorise implicitement le sens civique des réformateurs. Elle est révélatrice de l'ambivalence du discours réformateur, à la fois sincèrement scandalisé par la corruption, le clientélisme et les dysfonctionnements dans le gouvernement des villes, et plus ou moins subtilement xénophobe.

\section{Le fonctionnalisme de Merton : les machines avec les immigrés pauvres}

En 1949, au détour d'un développement sur l'analyse fonctionnelle en sociologie, Robert K. Merton avance l'idée que les machines ne sont pas « seulement » des entreprises de corruption organisée mais aussi des instruments d'intégration des immigrés ${ }^{48}$. Le propos de Merton est de distinguer les fonctions manifestes des fonctions latentes d'un phénomène social ${ }^{49}$. Les fonctions manifestes sont évidentes et recherchées par les acteurs; dans le cas des machines politiques, il s'agit du clientélisme, de la corruption, de l'intimidation des opposants, etc. Les fonctions latentes ne sont ni évidentes ni désirées. Merton soutient que l'intégration des immigrés est une conséquence non intentionnelle du phénomène clientélaire. Merton explique que le « contexte structurel» (la fragmentation du pouvoir politique local, la faiblesse de l'État central, le manque de coordination des différentes institutions politiques) ne permet pas de satisfaire les besoins

\footnotetext{
46. "L'homme idéalement adapté des pathologistes sociaux est "socialisé”. [...] Cela implique que l'homme adapté se conforme à la morale et aux buts bourgeois et "participe" à l'amélioration progressive des institutions respectables. [...] Sa mère et son père n’étaient pas divorcés, et son foyer n’était pas brisé. [...] Moins les traits et les "besoins" réalisés de l' "homme adapté" sont abstraits, plus ils gravitent autour des normes des bourgeois indépendants qui prétendent vivre selon les idéaux protestants dans les petites villes d'Amérique " (Mills (C. W.), « The Professional Ideology of Social Pathologists ", American Journal of Sociology, 49 (2), 1943, p. 180).

47. Cf. Banfield (E. C.), The Moral Basis of a Backward Society, Glencoe, The Free Press, 1956.

48. Cette dimension de la question n’avait pas échappé aux réformateurs. Dès 1933, H. Gosnell (« The Political Party versus the Political Machine », art. cité, p. 25-26) mentionne que le succès des machines repose sur leur capacité à venir en aide aux immigrés pauvres.

49. Merton (R. K.), Social Theory and Social Structure, Glencoe, The Free Press, 1949 (trad. fr. partielle : Élements de théorie et de méthode sociologique, Paris, Plon, 1965).
} 
des « sous-groupes aux besoins spécifiques » (les immigrés pauvres, les milieux d'affaires et le crime organisé). La machine est l'acteur qui va coordonner les différentes institutions du gouvernement urbain et remplir le vide structurel au niveau du pouvoir politique, avec des méthodes certes immorales et illégales, mais efficaces pour satisfaire les besoins des immigrés. La fonction latente de la machine est l'intégration des immigrés dans la société, l'économie et les institutions américaines.

L'analyse fonctionnelle de Merton influence un de ses collègues à Columbia, l'historien Richard Hofstadter. Dans The Age of Reform, considéré par Alan Brinkley comme « le livre le plus influent jamais publié sur l'histoire du $\mathrm{XX}$ siècle aux États-Unis » ${ }^{50}$, il révèle " les mobiles cachés des réformateurs » et décrit les boss comme des hommes du peuple, pragmatiques et affables ${ }^{51}$. Pour Hofstadter, les réformateurs sont des Américains « de souche » éduqués et installés qui voient à la fin du XIX ${ }^{e}$ siècle leur hégémonie sur la vie politique rétrécir de manière insupportable au profit des immigrés catholiques. Hofstadter ironise sur l'étroitesse d'esprit et l'angoisse de statut de ces réformateurs, qui cherchent « à s'accrocher à certaines valeurs de la vie agraire, à préserver l'initiative personnelle, l'opportunité individuelle et le type de personnalité qu'elles engendrent, et à maintenir une civilisation Yankee homogène ${ }^{52}$. Le travail de Hofstadter s'inscrit dans le contexte particulier du maccarthysme et la critique des réformateurs est une défense du liberal pluralism:

"Ébranlés par le souvenir du fascisme et la réalité du stalinisme, stupéfaits par le succès de Joseph McCarthy et d'autres démagogues, profondément inquiets de l'intolérance et des préjugés latents dans la vie politique, les intellectuels s'étaient engagés dans la défense de ce qui devint connu, selon l'expression heureuse de Arthur Schlesinger Jr., comme le "centre vital". La tâche des intellectuels selon eux était la défense des présupposés pluralistes de la démocratie américaine et la délégitimation des idéologies dangereuses qui les menaçaient à gauche et à droite ${ }^{53}$.»

Il faut noter que ni Merton ni Hofstadter ne s'intéressent spécialement aux machines. Merton est un sociologue de la science, n’a jamais travaillé sur les machines per se, et se sert de cet exemple pour illustrer l'analyse fonctionnelle avec un soupçon de provocation. Le livre de Hofstadter porte sur les mouvements progressistes et populistes au début du XXe siècle et n'aborde qu'en passant la question des machines. Quoi qu'il en soit, leur travail est un tournant historiographique. Merton permet un renversement de perspective : au lieu de décrire les boss comme des « amateurs arrogants » et les réformateurs comme

50. Brinkley (A.), « Richard Hofstadter's The Age of Reform: A Reconsideration », Reviews in American History, 13 (3), 1985, p. 462.

51. Teaford (J. C.), « Finis for the Tweeds and the Steffens... », art. cité.

52. Hofdtadter (R.), The Age of Reform..., op. cit., p. 28.

53. Brinkley (A.), « Richard Hofstadter's The Age of Reform... », art. cité., p. 463-464. 
des citoyens responsables épris de démocratie et d'idéal civique, il permet de penser l'analyse des machines en mettant en scène le boss comme l'allié des immigrés pauvres et les réformateurs comme des petits-bourgeois puritains et xénophobes ${ }^{54}$.

Les travaux sur les machines politiques de John M. Allswang dans les années 1970 sont caractéristiques de cette perspective. Allswang s'intéresse à la construction du boss ethnique (ethnic politician) ${ }^{55}$. Il montre comment les machines rendent des services aux électeurs en distribuant de la nourriture, des vêtements, des médicaments, c'est-à-dire en remplissant la fonction de welfare local. Elles rendent aussi des services immatériels comme l'intercession auprès d'un propriétaire exigeant ou d'un commissaire de police ; elles apportent une aide juridique aux électeurs qui veulent faire venir leur famille aux ÉtatsUnis. Allswang note même que les machines intelligentes sont celles qui ont su évoluer avec leur clientèle, en faisant du welfare au début du XX $\mathrm{XX}^{\mathrm{e}}$ siècle pour les immigrés pauvres, puis en subventionnant les petites entreprises de leurs enfants, comme à Chicago sous Daley ${ }^{56}$. Enfin, les politiciens des machines ne font pas que s'occuper des problèmes de la vie quotidienne. Ils défendent aussi les grandes idées de leurs électeurs, comme la liberté de l'immigration (combattue par les réformateurs au nom de la pureté de la nation américaine) et la liberté de la consommation d'alcool (contre les tendances prohibitionnistes de leurs adversaires de la classe moyenne protestante). Les programmes des machines reflètent les préoccupations de leur électorat sur l'indépendance de l'Irlande ou sur la reconstitution de la Pologne. L'historien Alexander Callow résume ainsi cette perspective :

« On peut critiquer le gaspillage, la corruption et l'inefficacité de la machine, mais si l'on considère les problèmes des pauvres - immigrés ou non -, les services offerts par un Tweed ou un Daley, la structure fragmentaire du gouvernement local, l'urbanisation rapide, les innovations technologiques, les buts du politicien de la machine et les idéaux du réformateur urbain, quelles étaient les alternatives viables au boss et à sa machine ${ }^{57}$ ? »

L'analyse fonctionnelle des machines suscite des critiques dans les années 1980. Elle est notamment accusée de souscrire à un liberal pluralism teinté de relativisme, complaisant envers la corruption des immigrés pauvres et associé à la célébration de la figure de Franklin D. Roosevelt en «boss de l'Amérique »,

54. Thelen (D.), « Urban Politics: Beyond Bosses and Reformers », Reviews in American History, 7 (3), 1979 , p. 407.

55. Allswang (J. M.), Bosses, Machines, and Urban Voters: An American Symbiosis, Port Washington (NY), Kennikat Press, 1977.

56. Stave (B. M.), Allswang (J. M.), McDonald (T. J.), Teaford (J. C.), «A Reassessment of the Urban Political Boss: An Exchange of Views », History Teacher, 21 (3), 1988.

57. Callow Jr (A. B.), « Review: Bosses, Machines, and Urban Voters: An American Symbosis by John M. Allswang ", American Historical Review, 83 (4), 1978, p. 1109. 
pragmatique et enclin à la redistribution des richesses, allié des classes populaires contre la petite-bourgeoisie protestante ${ }^{58}$. Mais ces reproches restent relativement secondaires par rapport à la littérature critique qui s'est développée dans les années 1970 contre la théorie fonctionnaliste.

\section{La critique progressiste des machines}

L'historiographie fonctionnaliste était favorable aux machines par opposition aux réformateurs. Mais à partir des années 1960 se développe une littérature critique nourrie par les références de la gauche intellectuelle des campus universitaires, proche du civil rights movement. La gauche intellectuelle considère que les boss rustres et corrompus présentent un degré de nuisance quasi équivalent à celui des démocrates ségrégationnistes du Sud. Pendant les «étés chauds » des années 1960, le boss de Chicago, Richard J. Daley, acquiert la détestation de la gauche intellectuelle pour s'être vanté, dans une conférence de presse, d'avoir ordonné aux policiers municipaux de tirer pour tuer (shoot to kill) sur tout manifestant s'apprêtant à jeter un cocktail Molotov (et donc susceptible de provoquer un incendie mortel).

Les travaux de Martin Shefter vont, par exemple, critiquer l'idée que les machines servent les immigrés en montrant que c'est la corruption qui est au principe de la centralisation (ou de la disparition) des premières machines, et non la défense, l'intégration ou la promotion sociale des immigrés irlandais ${ }^{59}$. Pour Shefter, la machine est une solution organisationnelle pour des politiciens confrontés à un triple problème : (1) contrôler un électorat instable : la croissance vertigineuse de la population et la diversité de l'immigration rendent les élections trop incertaines pour être décidées par la libre compétition politique ; (2) discipliner les politiciens locaux (ward politicians) rapaces en leur imposant à la fois une relation hiérarchique et un contrôle de leurs pratiques illégales ; (3) empêcher les milieux d'affaires de corrompre pour leur propre compte les fonctionnaires en dehors de tout contrôle et surtout de tout bénéfice pour l'organisation. L'analyse de Shefter montre les machines sous un jour nettement moins favorable que ne le fait l'analyse fonctionnelle. Elle participe d'une littérature critique qui redécouvre que les machines sont hostiles aux communistes et aux syndicats.

Les machines sont opposées au socialisme et au militantisme ouvrier parce que ceux-ci font peser le spectre d'un vote de classe, fondé sur des intérêts

\footnotetext{
58. Cf. DiGaetano (A.), « The Rise and Development of Political Machines. An Alternative to Merton's Functional Analysis ", Urban Affairs Quarterly, 24 (2), 1988 ; McDonald (T. J.), « The Problem of the Political in Recent American Urban History: Liberal Pluralism and the Rise of Functionalism ", Social History, 10, 1985.

59. Shefter (M.), «The Emergence of the Political Machine: An Alternative View », Theoretical Perspectives on Urban Politics, Englewoods Cliffs (NJ), Prentice Hall, 1976.
} 
matériels ou idéologiques ${ }^{60}$. Les machines sont structurellement hostiles aux politiques sociales à portée universelle, dans la mesure où celles-ci suppriment le principe clientélaire qui assure leur survie politique. Les machines ont intérêt à concevoir des politiques dont les bénéfices sont divisibles et dont la mise en œuvre est discriminante ${ }^{61}$. Les politiques qui bénéficient indistinctement à tous les habitants ne comportent aucune plus-value clientélaire : la lutte contre la pollution et pour une meilleure qualité de l'air, la lutte contre le jeu et la prostitution ne permettent pas au boss de récompenser des alliés et de punir des ennemis. Les machines contribuent donc à empêcher la formation d'un parti social-démocrate à l'européenne. De même, la rareté des ressources disponibles pour la distribution clientélaire implique de multiplier les emplois les moins bien payés, pour satisfaire le plus grand nombre d'électeurs, au détriment de la qualité des emplois. Erie estime que la tendance des machines irlandaises à générer beaucoup (jamais assez) d'emplois mal payés de policiers et de pompiers a nui à la mobilité sociale intergénérationnelle des Irlandais aux ÉtatsUnis $^{62}$. Les machines irlandaises ont également contribué aux problèmes de l'école publique dans les grandes villes, en limitant au maximum les financements publics, de façon d'une part à favoriser relativement l'école privée catholique (irlandaise) et d'autre part à punir les Juifs, qui forment le gros du corps enseignant ${ }^{63}$.

Les machines ont également nui à l'intégration économique et sociale des Noirs. Dans l'immense majorité des villes, les Noirs - qui pourtant avaient le droit de vote, surtout dans le Nord-Est - sont exclus de l'échange clientélaire ${ }^{64}$. Les Noirs sont privés des emplois municipaux stables ; les familles noires sont désavantagées par rapport aux immigrés européens qui ont pu s'appuyer sur les emplois clientélaires pour démarrer le processus de mobilité sociale intergénérationnelle. À Chicago, à une date aussi avancée que 1970, les Noirs représentent $40 \%$ de l'électorat mais seulement $20 \%$ des emplois municipaux; ces emplois sont toujours les plus pénibles et les moins bien payés ${ }^{65}$. Selon Katznelson et Grimshaw, la machine de Chicago s'assure du soutien de la classe politique noire en corrompant ses leaders, mais sans en faire bénéficier leurs électeurs ${ }^{66}$. Dans les grandes villes du Nord-Est dans les années 1960, les machines font construire les logements sociaux dans les quartiers noirs, de façon à concentrer les Noirs dans les ghettos, à protéger la pureté des quartiers de leurs clientèles,

60. Erie (S.), Rainbow's End..., op. cit.

61. Stone (C.), «Urban Political Machines... », art. cité.

62. Erie (S.), Rainbow's End..., op. cit.

63. Ibid.

64. Joyce (P. D.), «A Reversal of Fortunes. Black Empowerment, Political Machines, and City Jobs in New York City and Chicago », Urban Affair Review, 32 (3), 1997, p. 293.

65. Judd (D. R.), Swanstrom (T.), City Politics..., op. cit.

66. Katznelson (I.), Black Men, White Cities, New York, Oxford University Press, 1973 ; Grimshaw (W.), Bitter Fruit: Black Politics and the Chicago Machine, Chicago, University of Chicago Press, 1992. 
et à mieux contrôler le vote noir ${ }^{67}$. À Memphis, Ed Crump conquiert et garde le pouvoir en s'assurant du soutien de la communauté noire, ce qui est inhabituel dans une ville du Sud. Crump organise des barbecues dans les quartiers noirs de Memphis, et achète le vote noir avec des pastèques, du whisky et du coca-cola ${ }^{68}$. Sa machine paye la poll tax (l'impôt qui ouvre au droit de vote) des Noirs pauvres, d'une valeur de deux dollars par électeur (environ 50 dollars de 2009) ${ }^{69}$. La solidarité ethnique qui est au principe des machines s'est opérée au détriment des Africains américains.

En somme, des auteurs comme James C. Scott, Steven Erie ou Ira Katznelson pensent que les machines ont systématiquement empêché la formation de revendications de gauche, par les politiques qu'elles ont menées à la tête du gouvernement urbain, et par le fait de jouer les solidarités ethniques contre les solidarités sociales $^{70}$. Les machines ont privilégié des gains à court terme qui ont empêché les gains de long terme, c'est-à-dire la formation d'un « vrai » parti de gauche et ont donc contribué à l'absence d'un État providence de type européen.

\section{Conclusion}

Les machines politiques urbaines aux États-Unis ont disparu progressivement après la Deuxième Guerre mondiale. Le clientélisme, la corruption et surtout la domination des immigrés irlandais sur la vie politique municipale aux ÉtatsUnis ne pouvaient manquer de susciter une réaction parmi les classes moyennes protestantes, qui s'organisent pour reprendre le contrôle du gouvernement urbain. Le mouvement réformateur naît officiellement en 1894 avec la fondation de la National Municipal League, une association qui promeut un gouvernement urbain fondé sur l'expertise technique de professionnels indépendants du suffrage populaire. Des années 1890 à la Deuxième Guerre mondiale, les réformateurs vont œuvrer à changer les institutions locales et les règles du jeu électoral pour éliminer les machines et permettre aux classes moyennes protestantes de reprendre le contrôle des villes américaines. Les réformes consistent à lutter contre la fraude, à restreindre le droit de vote (pour empêcher les immigrés, les Noirs et les criminels de voter) et à modifier les règles du jeu électoral ${ }^{71}$.

67. Erie (S.), Rainbow's End..., op. cit.

68. Biles (R.), «Ed Crump versus the Unions... », art. cité.

69. Dowdy (G. W.), Mayor Crump Don't Like It: Machine Politics in Memphis, Jackson, University Press of Mississippi, 2006.

70. Scott (J. C.), «Corruption, Machine Politics and Political Change », American Political Science Review, 63 (4), 1969 ; Erie (S.), Rainbow's End..., op. cit. ; Katznelson (I.), City Trenches: Urban Politics and the Patterning of Class in the United States, New York, Pantheon, 1981.

71. Cf. Banfield (E. C.), Wilson (J. Q.), City Politics, op. cit.; Gosnell (H.), « The Political Party versus the Political Machine », art. cité ; Murphy (R. D.), « Politics, Political Science, and Urban Governance: A Literature and a Legacy », Annual Review of Political Science, 5, 2002 ; Bridges (A.), Kronick (R.), "Writing the Rules to Win the Game: The Middle-Class Regimes of Municipal Reformers », Urban Affairs Review, 34 (5), 1999. 
Ce mouvement de réforme institutionnelle s'inscrit dans un contexte d'évolution profonde de la société américaine. D’abord, les lois de 1921 et 1924 sur les quotas d'immigration puis la crise économique réduisent la proportion des populations immigrées et donc l'électorat naturel des machines. La stabilisation des électorats a favorisé l'émergence d'une compétition politique plus lisible qui a été défavorable aux machines ${ }^{72}$. Ensuite, la mise en ouvre du New Deal par Franklin D. Roosevelt bouleverse les mécanismes de protection sociale et d'allocation des emplois publics. Si certains boss parviennent à monnayer leurs services dans les swing states, la grande majorité sont concurrencés par la formation d'un État providence national dont les bénéfices sont alloués de manière bureaucratique et non discrétionnaire. Enfin, l'embourgeoisement des groupes ethniques blancs après la Deuxième Guerre mondiale et la migration intérieure de plus de cinq millions de Noirs ruraux du Sud vers les villes industrielles du Nord-Est provoquent le white flight, la sécession des Blancs urbains vers les banlieues résidentielles (suburbs). L'arrivée des Noirs dans les centresvilles fait craindre aux Blancs une dépréciation des prix de l'immobilier, une baisse de la qualité des écoles et une augmentation de la criminalité. Simultanément, les banlieues résidentielles deviennent plus accessibles, tant du point de vue financier que de celui des transports. Le white flight est par nature un élément destructeur de l'activité des machines, puisqu'il sépare l'électeur de son precinct captain et disperse les populations dans de vastes banlieues qu'il est bien plus ardu d'organiser politiquement ${ }^{73}$. Les électeurs noirs, galvanisés par le civil rights movement, n'imaginent pas élire des politiciens irlandais au niveau local. Pendant la période de transition des années 1950 et 1960, les démocrates essaient de faire subsister les machines, mais ces dernières sont devenues des organisations vieillissantes, à l'exception notable de Chicago ${ }^{74}$.

Dans ce travail, on a tracé les contours d'une forme spécifique d'ancrage social des partis politiques, à travers le cas des machines politiques aux ÉtatsUnis ; on a également resitué le contexte du débat historiographique autour des machines. Dans son aspect le moins intéressant, ce débat historiographique peut verser dans une discussion normative sur le jugement que le chercheur doit porter sur les machines, voire dans un procès d'intention sur les arrièrepensées des auteurs impliqués. Ainsi Andrew Greeley, un sociologue de l'immigration irlandaise, accuse Steven Erie, l'auteur d'origine irlandaise de Rainbow's End, de «haine de soi » (self-hatred) parce qu'il décrit la corruption des machines irlandaises ${ }^{75}$. On retrouve ce type de débats dans la littérature italienne

72. Cf. la revue de littérature de Boulay (H.), DiGaetano (A.), « Why Did Political Machines Disappear? », art. cité.

73. Banfield (E. C.), Wilson (J. Q.) (City Politics, op. cit.) notent également que le développement des médias de masse et de la communication politique audiovisuelle n'a pas pu bénéficier aux machines.

74. Sur Chicago et Richard J. Daley, cf. Royko (M.), Boss..., op. cit.

75. Greeley (A.), « Review: Rainbow's End », Contemporary Sociology, 19 (1), 1990, p. 66-68. 
contemporaine sur le clientélisme, qui avance une perspective assez radicalement constructiviste en analysant le concept de « clientélisme » comme une catégorie de délégitimation de pratiques politiques construites comme déviantes $^{76}$. L'entreprise est parfaitement légitime mais peut devenir elle-même assez normative. Cesare Mattina fustige, par exemple, le "moralisme stigmatisant » de la recherche américaine et le "syndrome de l'autoflagellation permanente » chez les chercheurs italiens qui travaillent sur l'élucidation du « retard démocratique » de l'Italie, tandis que Simona Piattoni insinue que le mépris (réel) de Banfield et Wilson pour les machines politiques aux États-Unis proviendrait de leur racisme (introuvable) envers « les nouveaux immigrants en provenance de pays latins ${ }^{77}$. On peut faire à cette démarche deux objections.

D’une part, quelles que soient les arrière-pensées des uns ou des autres, les machines américaines restent caractérisées par la corruption. La réalité historique ne change pas en fonction du degré de radicalité du constructivisme qui préside à la réappréciation du phénomène clientélaire. Comme dirait Bourdieu, « le monde social, avec ses hiérarchies qui ne se laissent pas si facilement relativiser, n'est pas relativiste ${ }^{78}$. D'autre part, le cas des machines montre que la problématique du clientélisme recèle un potentiel heuristique qu'il serait dommage de réduire à des questionnements normatifs. Le travail de Steven Erie montre, par exemple, comment le clientélisme n'est qu'une composante d'une stratégie de groupe de mobilité intergénérationnelle s'appuyant sur la monopolisation des emplois publics. Le livre de Roger Waldinger (Still the Promised City?) analyse les stratégies d'intégration sur le marché du travail des différents groupes immigrés à New York en mobilisant le concept de "niche ethnique ", c'est-à-dire de secteurs d'emploi dont l'accès est contrôlé par des mécanismes de solidarité ethnique ${ }^{79}$. Dans cette perspective, l'allocation clientélaire des emplois publics par le contrôle de la bureaucratie urbaine n'est qu'un moyen comme un autre pour un groupe de se constituer un secteur qui sert de tremplin dans la société d'accueil. Dans les années 1960-1970, ce sont les Noirs qui prennent le relais des Irlandais pour les emplois publics municipaux, notamment par le biais des programmes de discrimination positive, permettant ainsi la constitution d'une classe moyenne noire. Malheureusement pour les Noirs, la crise fiscale urbaine des années 1970 va mettre à mal cette stratégie.

\footnotetext{
76. Sur le clientélisme comme catégorie savante de délégitimation, cf. notamment Briquet (J.-L.), « La politique clientélaire... ", art. cité.

77. Mattina (C.), La régulation clientélaire. Relations de clientèle et gouvernement urbain à Naples et à Marseille (1970-1980), Thèse de doctorat en science politique, Institut d'Études politiques de Grenoble, 2003, p. 67 et p. 58 ; Piattoni (S.), « Le clientélisme revisité. Politique clientéliste et développement économique dans l'Italie d'après-guerre ", Pôle Sud, 19, 2003, p. 156. Banfield et Wilson ne parlent pas des Italiens dans City Politics.

78. Bourdieu (P.), Méditations pascaliennes, Paris, Seuil, 1997, p. 11.

79. Waldinger (R.), Still the Promised City? New Immigrants and African-Americans in Post-Industrial New York, Cambridge (MA), Harvard University Press, 1996.
} 
Enfin, il reste aux sciences sociales à investiguer les nouvelles modalités de la relation clientélaire, dans le sillage des travaux de Nicole Marwell ${ }^{80}$. Marwell montre comment la privatisation et la décentralisation de l'État providence pendant les années Reagan a permis la mise en place d'un " échange triadique »: les autorités politiques locales financent des associations caritatives pour fournir des politiques sociales aux populations défavorisées ; les associations font voter ces populations pour les politiciens qui les financent, de manière à assurer la continuité des politiques sociales et la pérennité organisationnelle des associations. En somme, l'analyse du clientélisme peut (toujours) aller bien au-delà des questions normatives et nous renseigner sur les questions centrales de pouvoir et d'inégalités.

François Bonnet est Assistant Professor au département de sociologie et d'anthropologie de I'Université d'Amsterdam. Ses travaux portent sur les questions urbaines et la production de l'ordre social. II a notamment publié "Un crime sans déviance : le vol en interne comme activité routinière ", Revue française de sociologie, 49 (2), 2008 ; " La distance sociale dans le travail de terrain : compétence stratégique et compétence culturelle dans l'interaction d'enquête ", Genèses, 4, 2008, et "Managing Marginality in Railway Stations: Beyond the Welfare and Social Control Debate ", International Journal of Urban and Regional Research, 33 (4), 2009).

bonnet@uva.nl

80. Marwell (N.), « Privatizing the Welfare State: Nonprofit Community Based Organizations as Political Actors ", American Sociological Review, 69 (2), 2004 ; et, du même auteur, Bargaining for Brooklyn. Community Organizations in the Entrepreunarial City, Chicago, University of Chicago Press, 2007. 